\title{
Caregiver Tele-Assistance for Reduction of Emotional Distress During the COVID-19 Pandemic. Psychological Support to Caregivers of People with Dementia: The Italian Experience
}

\author{
Emanuela Rotondo $^{\mathrm{a}}$, Daniela Galimberti ${ }^{\mathrm{a}, \mathrm{b}, *}$, Matteo Mercurio $^{\mathrm{a}}$, Giulia Giardinieri ${ }^{\mathrm{a}}$, Sara Forti ${ }^{\mathrm{a}}$, \\ Roberto Vimercati $^{\mathrm{a}}$, Vittoria Borracci ${ }^{\mathrm{a}}$, Giorgio G. Fumagalli ${ }^{\mathrm{a}}$, Anna M. Pietroboni ${ }^{\mathrm{a}}$, \\ Tiziana Carandini ${ }^{\mathrm{a}}$, Alessandro Nobilic ${ }^{\mathrm{c}}$, Elio Scarpini ${ }^{\mathrm{a}, \mathrm{b}}$ and Andrea Arighi ${ }^{\mathrm{a}}$ \\ a Fondazione IRCCS Ca' Granda, Ospedale Policlinico, Milan, Italy \\ ${ }^{\mathrm{b}}$ University of Milan, Milan, Italy \\ ${ }^{\mathrm{c}}$ Istituto di Ricerche Farmacologiche Mario Negri IRCCS, Milan, Italy
}

Accepted 28 October 2021

Pre-press 15 November 2021

\begin{abstract}
.
Background: COVID-19 pandemic worsened vulnerability of patients with dementia (PWD). This new reality associated with government restriction and isolation worsened stress burden and psychological frailties in PWD caregivers.

Objective: To give tele-psychological support to caregivers and evaluate the effect of this intervention by quantifying stress burden and quality of life during the first COVID-19 lockdown.

Methods: 50 caregivers were divided into two groups: "Caregiver-focused group" $(\mathrm{Cg})$ and "Patient-focused group" $(\mathrm{Pg})$. Both groups received telephone contact every 2 weeks over a 28 -week period, but the content of the call was different: in $\mathrm{Cg}$, caregivers answered questions about the state of the PWD but also explored their own emotional state, stress burden, and quality of life. In Pg instead, telephone contacts were focused only on the PWD, and no evaluation regarding the caregiver mood or state of stress was made. Psychometric scales were administered to evaluate COVID-19 impact, stress burden, and quality of life.

Results: Considering the time of intervention, from baseline (W0) to W28, Zarit Burden Interview and Quality of Lifecaregiver questionnaires remained unchanged in $\mathrm{Cg}$ as compared with baseline $(p>0.05)$, whereas they worsened significantly in Pg $(p<0.01)$, showing increased stress over time and decreased quality of life in this group. Moreover, Impact on Event Scale values improved over the weeks in $\operatorname{Cg}(p=0.015)$, while they remained unchanged in $\operatorname{Pg}(p=0.483)$.

Conclusion: Caregivers who received telephone support about their mood and stress burden did not worsen their psychological state during the time of intervention, as did instead those who did not get such support.
\end{abstract}

Keywords: Caregiver, COVID-19 pandemic, people with dementia, quality of life, stress burden, tele-psychological support

\footnotetext{
*Correspondence to: Daniela Galimberti, University of Milan, Fondazione IRCCS Ca' Granda, Ospedale Policlinico, Milan, Italy. Tel.: +39 02 55033847; Fax: +39 02 55036580; E-mail: daniela. galimberti@unimi.it; daniela.galimberti@policlinico.mi.it.
}

\section{INTRODUCTION}

Currently, more than 50 million people have dementia worldwide [1]. Dementia is associated with varying degrees of cognitive decline and behavioral changes, which have a huge impact on the quality 
of life of persons affected and their relatives. During normal times, people with dementia (PWD) are very vulnerable and dependent on family or professional caregivers in everyday life.

The 2019 new coronavirus disease (COVID-19) was declared a "Public Health Emergency of International Concern" (PHIC) on January 30, 2020, and the World Health Organization (WHO) declared the "COVID-19 pandemic" on March 11, 2020 [2].

This COVID-19 pandemic worsened vulnerability of PWD directly: a diagnosis of dementia is in fact an important risk factor for mortality in patients with COVID-19 [3]. Many countries have implemented strict public health measures to protect vulnerable groups such as PWD from the deleterious physical effects of COVID-19. In Italy, the first strict lockdown lasted almost 3 months (March-May 2020) with severe restrictions in daily living habits.

Psychological changes become evident after the introduction of the restrictions applied by the Government during the COVID-19 pandemic, such as social distancing and limitations in individual mobility and social activities. These restrictions may have had negative consequences on the possibility of being psychologically and emotively supported by others; low social support has been associated with higher depression in caregivers [4].

During this period, caregivers might have experienced higher than usual stress and burden because communication and coordination with healthcare providers was difficult, thus they were unable to rely on their usual network of formal and informal in-home supports, face escalating challenges in accessing needed in-home care. Additionally, support programs, such as adult day health care, may not be available. Caregivers may also experience negative physical and mental health outcomes. Many caregivers experienced feelings of social isolation, which may be exacerbated by social distancing policy measures. Older caregivers may be at particular risk for injury as they take on additional hands-on caregiving responsibilities, such as assisting with mobility and transfers in the absence of other help. Added caregiver burden and lack of informal care prevented older care recipients from being safely maintained in their homes, increasing the risk for requiring care in emergency departments, hospitals, or long-term care facilities [5].

In this new reality, caregivers often experienced high burden that negatively compromised their mental wellbeing, as a result of the loneliness brought about by the confinement and isolation. This has been associated with health problems such as compassion fatigue that force some family caregivers to relinquish care roles of their loved ones to long-term care $[6,7]$.

Isolation had negative effects in about half of PWD, also for the discontinuation of essential services for their care [8,9]. Prolonged time of isolation had negative effects on caregivers, as demonstrated by higher levels of depression and anxiety [10]. Educational level appears to be a protective factor. Women seem to suffer the consequences of the lockdown on anxiety, depression, and stress more than the men [11].

Quarantine induced a rapid increase of behavioral and psychological symptoms of dementia (BPSD) in approximately $60 \%$ of patients and stress-related symptoms in two-thirds of caregivers. An increased prevalence of symptoms of anxiety, feeling of helplessness, and anguish were reported by caregivers [8] Increased concerns for patient health and increased familial conflicts were also reported [8]. Presence of housemates reduced the risk of depression and conflicts, thus indicating that caregiver burden may be mitigated by contrasting loneliness and supporting needs of caring with a network of helps. There seems to be an association between psychological symptoms of anxiety and depression in caregivers and increased BPSD burden [8-12].

The COVID-19 pandemic is unmasking an emerging form of technology-related social inequalities that become a major factor of vulnerability. A series of political and community interventions aiming to solidify the social safety net is needed to support the most socially vulnerable population and prevent increasing both their vulnerability to the pandemic and the social health inequalities [13, 14].

Considering all the above, the aim of this study was to: 1) give tele-psychological support to caregivers and evaluate the effect of this intervention by quantifying stress burden and quality of life during COVID-19 lockdown period (from April to November 2020), 2) evaluate psychological impact of COVID-19 pandemic in caregivers of PWD.

\section{METHODS}

\section{Participants}

Caregivers were recruited through local database collected in Neurodegenerative Disease Unit of the Fondazione IRCCS Ca' Granda Ospedale Maggiore Policlinico, Milan. Recruitment has been carried out from April to May 2020 and involved caregivers of PWD. Patients and their caregivers have had a follow 
up visit at our outpatient service over the last year before the pandemic. The study was approved by the Local Ethics Committee ('Milano - Area 2', number 362_2020 - April 30th, 2020).

Participants' inclusion criteria were: A) be PWD caregiver, cohabitant or not, B) give oral informed consent, C) age $>18$ years, D) understand and communicate in Italian language, E) be able to understand and answer the questions, F) spend 8 hours or more per week with PWD (before COVID-19 pandemic lockdown).

One hundred and nine caregivers were contacted by phone, and 50 of them fulfilled inclusion criteria and accepted to participate.

All participants had a first telephone contact with a psychologist. At baseline (Week 0 - W0) the following tests were administered:

- COVID-19 questionnaire: a new questionnaire was created to explore caregiver changes during national lockdown for COVID-19 infection. The questionnaire included: 1) sociodemographic information about caregiver who completed (i.e., town, age, job), 2) clinical information about relatives PWD (i.e., relationship, diagnosis, type and level of disease), 3) daily habits related with PWD (i.e., weekly hours spent together, help in care management, free time, emotional state, mood, communication skills). Issues included in point 3 ) were analyzed before (using retrospective questions) and during COVID-19 lockdown. Notably, the questionnaire has not been developed as a quantitative scale to be validated but as an instrument to get relevant information over a phone call duration.

- Neuropsychiatric Inventory (NPI) [15]

- Zarit Burden Interview (ZBI) [16]

- Quality Of Life caregiver (QOL- cg) [17]

- Impact of Event Scale - revised (IES) [18].

After this first step, participants were randomly divided into two groups: "Caregiver-focused group" (Cg: psychological telephone contacts with interview about PWD, exploring also emotional state, stress burden, and quality of life of caregiver) and "Patient-focused group" (Pg: psychological telephone contacts with interview about PWD, no evaluation about caregiver mood or state of stress). Randomization was performed using the random function of Microsoft Excel. Diagnoses at inclusion were as follows: 26 patients had Alzheimer's disease (Cg/Pg: 11/15), 4 frontotemporal dementia (Cg/Pg:
2/2), 6 Lewy body dementia ( $\mathrm{Cg} / \mathrm{Pg}: 3 / 3), 3$ primary progressive aphasia $(\mathrm{Cg} / \mathrm{Pg}: 2 / 1), 9$ other dementias (vascular dementia or mixed dementia; $\mathrm{Cg} / \mathrm{Pg}: 5 / 4$ ). There were no significant differences in terms of frequency of diagnoses between $\mathrm{Cg}$ and $\mathrm{Pg}$.

Telephone contacts $(15 \mathrm{~min})$ were made every two weeks over a 28-week period (from April to November 2020) by trained clinical psychologists. Assessment at Week 28 (W28) were done using: ZBI, QOL-cg, IES, and questionnaire about telephone contact satisfaction and usefulness.

At 32 weeks (Week 32- W32) (within December 2020) a further follow up was done including ZBI and QOL-cg (Fig. 1).

\section{Statistical analysis}

The data obtained from the study were analyzed using statistical analysis software Jamovi (https://www.jamovi.org/). Frequency and percentage were used for categorical data, whereas mean and standard deviation values were used for continuous data. Chi-square test was used for the analysis of categorical data, and $t$ student and repeated measures ANOVA were used in the analysis of continuous data. Values with $p<0.05$ were considered statistically significant.

\section{RESULTS}

Fifty participants were randomly assigned into two groups: 25 in $\mathrm{Cg}$ and 25 in $\mathrm{Pg}$. After the random distribution, 2 caregivers in $\mathrm{Cg}$ refused to continue, therefore a total of 48 participants (33 women, 15 men) agreed to participate in the study: 23 were in $\mathrm{Cg}$ and 25 in $\mathrm{Pg}$.

Demographics and details of the population are reported in Tables 1 and 2. No significant differences in age, sex distribution, and schooling were found between participants' groups. Regarding the cognitive state of PWD, mean value of Mini-Mental State Examination (MMSE) [19] in patients followed by Cg caregivers was 17.4, whereas in PWD followed by caregivers of the Pg group was $18.4(p>0.05)$. Time from diagnosis, age, and schooling of PWD were also not statistically different $(p>0.05)$.

Thirteen out of 48 caregivers dropped out during the study (6/23 in $\mathrm{Cg}$ and $7 / 25$ in $\mathrm{Pg}, p=0.882)$. Reasons were multiple: personal problems (work or smart-working for $8 / 10$ hour a day, major changes in family routine, hospitalization for illness) and troubles about patients (aid reduction in patient care and assistance, inside and outside home). In none of the 


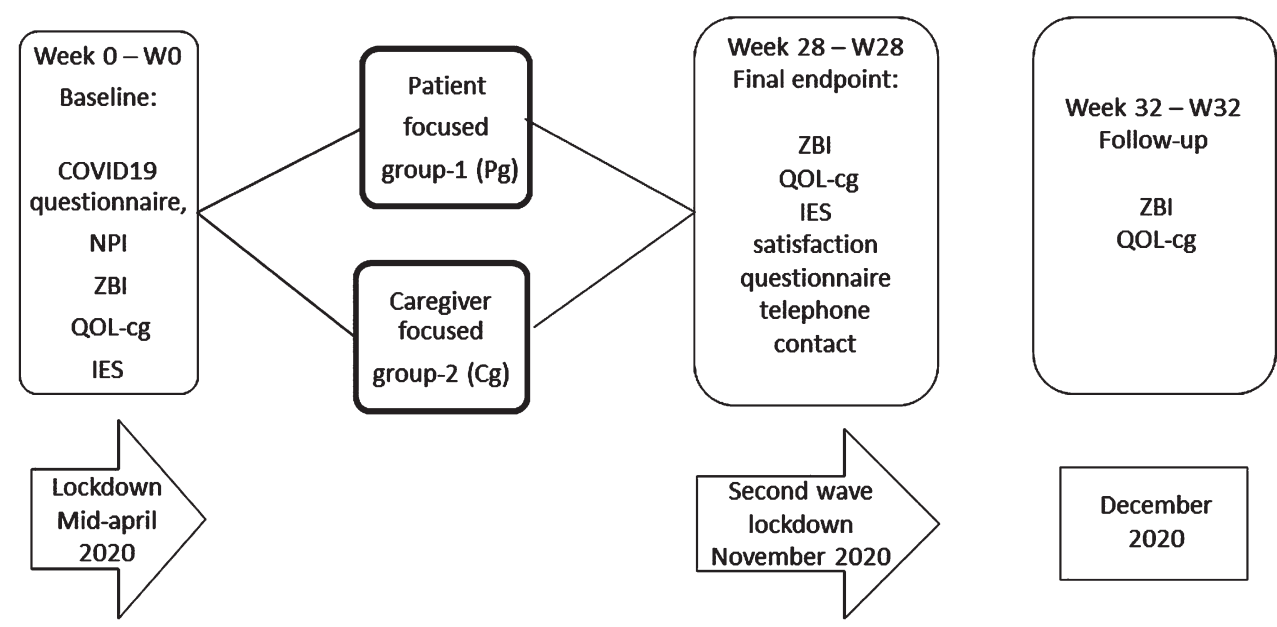

Fig. 1. Study timeline and pandemic situation in Italy.

Table 1

Demographic characteristics of participants and comparison between the two groups

$(\mathrm{Cg}$ versus $\mathrm{Pg}$ ) using $t$-test analysis and Chi-square test

\begin{tabular}{lccc}
\hline & Cg $(23)$ & $\operatorname{Pg}(25)$ & $\mathrm{p}$ \\
\hline Age $(\mathrm{y}$, mean \pm SD) & $67.8 \pm 12.79$ & $61.2 \pm 18.47$ & 0.195 \\
Education $(y$, mean \pm SD) & $12.2 \pm 3.99$ & $12.9 \pm 4.99$ & 0.616 \\
Gender $(\mathrm{M}: \mathrm{F})$ & $7: 16$ & $10: 15$ & 0.489 \\
\hline
\end{tabular}

$\mathrm{SD}$, standard deviation.

Table 2

Characteristics of PWD and comparison between the two groups (Cg versus Pg) using $t$-test analysis and Chi-square test

\begin{tabular}{llccc}
\hline & & $\mathrm{Cg}(23)$ & $\operatorname{Pg}(25)$ & $p$ \\
\hline \multicolumn{1}{c}{ Mini-Mental State Examination (mean \pm SD) } & $18.4 \pm 7.21$ & $17.4 \pm 6.58$ & 0.606 \\
\hline Years of disease & $1-3$ & 6 & 6 & 0.868 \\
& $4-6$ & 10 & 11 & 0.971 \\
\multirow{5}{*}{ Diagnosis } & $7-10$ & 4 & 5 & 0.817 \\
& $>10$ & 3 & 3 & 0.913 \\
& AD & 11 & 15 & 0.398 \\
& FTD & 2 & 2 & 0.931 \\
Patient Impairment & LBD & 3 & 3 & 0.913 \\
& PPA & 2 & 4 & 0.502 \\
& Other & 5 & 4 & 0.611 \\
& Cognitive & 23 & 25 & 1.00 \\
& Anosognosia & 10 & 6 & 0.153 \\
Level of cognitive impairment & Impact in daily living & 18 & 15 & 0.173 \\
& Behavioral & 14 & 14 & 0.732 \\
& Mild & 9 & 8 & 0.606 \\
& Moderate & 7 & 11 & 0.332 \\
& Severe & 7 & 5 & 0.404 \\
& Bedridden & 0 & 1 & 0.332 \\
\hline
\end{tabular}

$\mathrm{SD}$, standard deviation.

cases was the drop out due to COVID development in patients or caregivers.

To evaluate the evolution of stress burden (using ZBI) and quality of life (using QOL-cg), we compared questionnaire values at timepoints $\mathrm{W} 0$ and W28 (intervention) and follow-up W28-W32 (no intervention) in each group using repeated measures ANOVA. To evaluate pandemic impact, we compared IES questionnaire at timepoints W0 and W28 using repeated measures ANOVA.

Considering the time of intervention, from baseline (W0) to W28, ZBI and QOL-cg questionnaires 

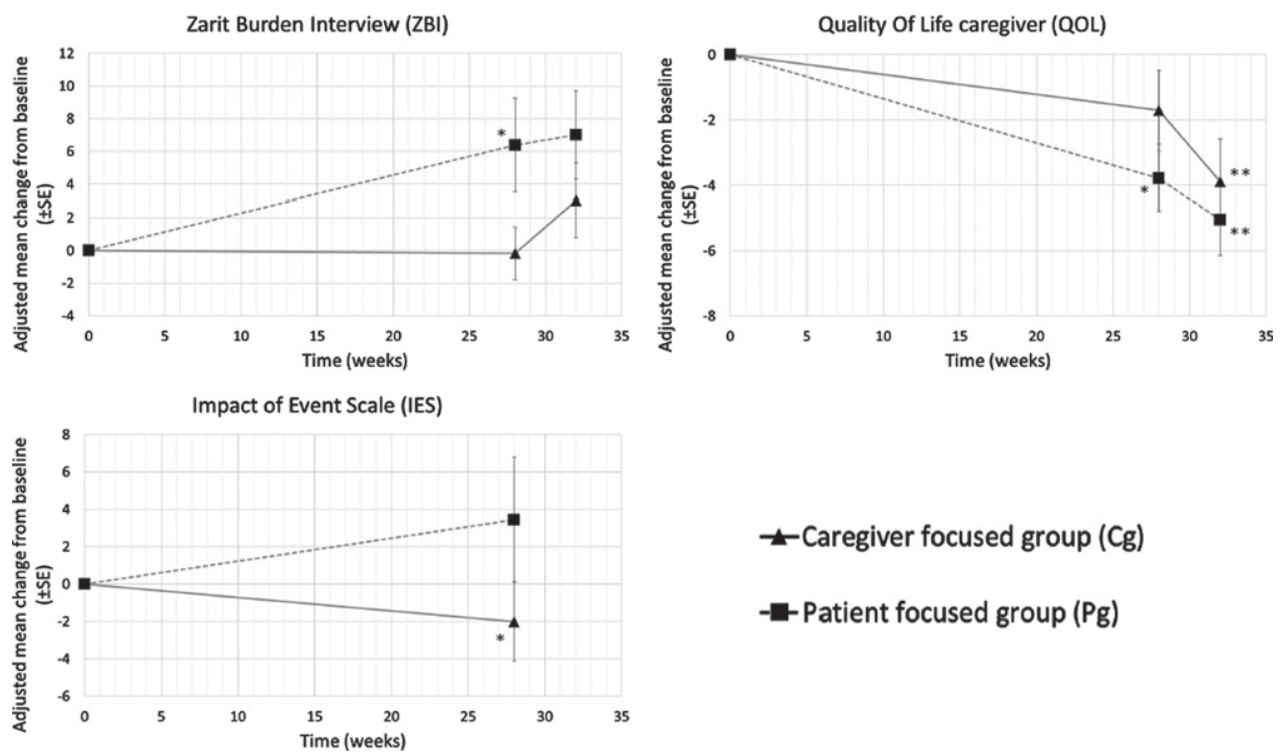

\section{$\mp$-Caregiver focused group $(\mathrm{Cg})$ \\ Patient focused group $(\mathrm{Pg})$}

Fig. 2. ZBI, QOL-cg, and IES questionnaires adjusted mean change from baseline in Pg and Cg at timepoints W0-W28-W32 (initial values of patient were subtracted from each value before obtaining the mean). IES questionnaire comparison between Pg and Cg groups at timepoints W0-W28 using repeated measures ANOVA. $p$ values refer to comparisons in the same group over time. * $p$ values: W28 versus baseline; $* * p$ values: W32 versus W28.

remained unchanged in $\mathrm{Cg}$ as compared with baseline (ZBI: $p=1$, QOL-cg: $p=0.108$ ), whereas worsened significantly in $\operatorname{Pg}(p=0.017$ and $p<0.001$, respectively, Fig. 2), showing increased stress over time and decreased quality of life in this group. Moreover, IES values improved over the weeks $(p=0.015)$ in $\mathrm{Cg}$, while remained unchanged in $\operatorname{Pg}(p=0.483$, Fig. 2).

At follow up (W32-no intervention-as compared with W28), ZBI and QoL worsened in both groups, with a marked increase of ZBI in Cg (Fig. 2), although the statistical threshold was not reached. Instead, QoL was significantly changed $(p=0.029$ in $\mathrm{Cg}$ and $p=0.025$ in Pg, Fig. 2).

All caregivers ( $\mathrm{Pg}$ and $\mathrm{Cg}$ ) who were available to complete the study (32 weeks) appreciated this psychological telephone contacts and the opportunity to speak directly with a psychologist [35/35]. In $\mathrm{Cg}$, satisfaction was 8.9/10 and in $\operatorname{Pg} 8.4 / 10(p=0.134)$. Regarding psychological telephone contacts content, $\mathrm{Cg}$ give a higher satisfaction score $(8.5 / 10$, about COVID-19 7.76/10) than $\operatorname{Pg}(7.1 / 10, p<0.001$ and about COVID-19 6.2/10, $p<0.05$ ).

\section{DISCUSSION}

The present study aimed, firstly, at investigating levels of stress burden and quality of life in caregivers of PWD during the 2020 lockdown due to the COVID-19 pandemic and support them through psy- chological telephone contacts (telephonic counseling sessions every two weeks) and secondly to evaluate the psychological impact of COVID-19 pandemic in caregivers of PWD.

We showed that caregivers who received psychological telephone support about their mood and stress burden $(\mathrm{Cg})$ did not worsen over time from the beginning of restrictions, and gave the same values at questionnaires. Conversely, caregivers contacted just to ask the health conditions of PWD (Pg) worsened. Therefore, psychological telephone intervention is an important instrument to support caregiver in stress management and perception of their quality of life. The ability to express their mood, be heard, and feel understood could be very helpful for people that every day spend time, energy, and emotion with PWD. Therefore, we hypothesize that introducing a direct communication through psychological telephone contacts and engaging caregivers about their distress would be beneficial, not only in in caregivers of PWD but possibly also in those of other irreversible conditions. This approach, easy and cheap, could be included in clinical settings of Neurodegenerative Disease Units, or in general in all Clinical Hospital Units. Notably, the severity of dementia of patients followed by caregivers in both groups was similar, thus avoiding any bias due to the cognitive state of PWD. However, no details were available on different (behavioral or not) aspects, which could 
influence caregivers' stress burden, such as aggressiveness, impulsiveness, hypersexuality, or insomnia.

Of notice, at follow up, QoL worsened in both groups as compared with the end of the intervention, suggesting that any kind of support may have a beneficial effect on caregivers. Nevertheless, the most striking change was observed in $\mathrm{ZBI}$ in $\mathrm{Cg}$, that was unchanged over the time of intervention and increased suddenly right after the end on the study, suggesting a detrimental effect in Cg after stopping the psychological support.

We acknowledge that the follow up has been done for four weeks only, thus no definitive results can be ruled out. However, the trend of abrupt worsening in Cg suggests that, in view of a longer beneficial effect, the intervention should be continuative over time, possibly with different timing (i.e., every four weeks instead of two) for a matter of feasibility in terms of time and costs.

The COVID-19 pandemic has forced dramatic changes in medical and psychological care delivery. Safety concerns regarding viral transmission have led to restrictions in face-to-face clinical encounters and increased use of telehealth approaches $[20,21]$. Alzheimer Europe recommends the following for PWD and their caregivers: building a support network, keeping well informed, guaranteeing food and medical supplies, enjoying leisure activities, staying physically and mentally active, and keeping socially connected [22]. In the age of information and communications technology, technology home-based interventions [23] (smartphones, tablets, computers, smart televisions, virtual assistants, ambient assistive devices, etc.) can support most of the above recommendations; they facilitate information sharing and online shopping, provide access to entertainment, increase social connectedness, and, most importantly, these interventions enable the distribution of health-related information and services. Telehealth is a potential major tool to deliver routine health care and prevent the risk of viral exposure, especially for people at higher risk [24, 25]. Numerous studies conducted in the recent years have reported that informal/family dementia caregivers can access Internet-based interventions, and they received positive effects on their wellbeing [28-32]. The COVID-19 pandemic is unmasking an emerging form of technology-related social inequalities: political and community interventions are needed to support the most socially vulnerable population and prevent social health inequalities. We choose a direct communication using telephone, bypassing caregiver inability to use tablet or PC and internet connection difficulties [13]. Our results underline the need of caregivers to be supported in the patient care, particularly in critical situations related to COVID-19 pandemic.

Given the opportunity to speak and be heard directly by a health worker (in this case a psychologist) makes the caregivers feel closer and supported by the health facilities. Thus, if the content of this contact is supportive from a psychological point of view (talking about stress, emotional state), it improves subjective satisfaction and gives the caregiver support to control the load of stress and improve the quality of life (as shown by changes in ZBI and QOL-cg, respectively). Similarly, the adaptability to the impact of the pandemic situation in daily life during lockdown months resulted higher; the $\mathrm{Cg}$ group seems to be better adapted to the impact of the pandemic situation in daily life during lockdown months. Otherwise, in $\mathrm{Pg}$, stress burden increased and quality of life got worse, although the COVID-19 pandemic impact did not apparently change over the period of study.

We are aware that the COVID-19 pandemic is a component out of our control, and it might have affected the caregiver answers to the questionnaires. On the other hand, based on our data, the lockdown impact was not the main worry of caregivers, although it was a variable that worsened an already existing frail situation. For this reason, independent of the COVID, we hypothesize that the opportunity to have a psychological telephone contacts could be beneficial to all caregivers, i.e., not only in this emergency, but also in clinical routine. Stress burden and quality of life remained unchanged despite the pandemic situation in caregivers who received the telephone support (focused on patient and own psychological situation). In line with this, data published in the pre-COVID era suggest that interventions delivered via telephone and computer have the potential to augment existing dementia care, particularly those that provide caregivers with access to practical strategies to manage care of PWD and their own wellbeing, advice and support from peers and/or clinicians [33].

In conclusion, in our study we followed a group of caregivers for 32 weeks ( 28 weeks of treatment and 4 weeks of follow-up), a long period in a critical period of COVID-19 pandemic, and gave them substantial support. We tested psychological intervention using telephone contact and demonstrated it to be very useful to overcome the restrictions. We think our results underline the importance of telepsychological support, but further studies in larger 
cohorts would be needed to confirm our preliminary data. Unfortunately, in this study the average of drop out was high. Nevertheless, this would be hard to avoid in a population with a considerable burden of activity, linked to its own life and PWD care out of our control. In addition, it cannot be excluded that the sample participating in the study was not biased by other variables (number of family members, social condition, economic status. etc.), therefore confirmatory future studies on larger and better-defined populations would be needed.

\section{ACKNOWLEDGMENTS}

This work was supported by grants from the Italian Ministry of Health (Ricerca Corrente), Dino Ferrari Center and Fondazione Gigi \& Pupa Ferrari Onlus. VB is supported by the Italian Ministry of Health, grant RF-2018-12365333.

Authors' disclosures available online (https://www.j-alz.com/manuscript-disclosures/215185r2).

\section{REFERENCES}

[1] Alzheimer's Disease International (2019) World Alzheimer Report 2019. Attitudes to dementia. Alzheimer's Disease International, London, UK.

[2] World Health Organization (2020) Novel Coronavirus (2019-nCoV): Situation Report, 12. Available online at: https://apps.who.int/iris/handle/10665/330777 (accessed June 15, 2020).

[3] Bianchetti A, Rozzini R, Guerini F, Boffelli S, Ranieri P, Minelli G, Bianchetti L, Trabucchi M (2020) Clinical presentation of COVID-19 in dementia patients. $J$ Nutr Health Aging 24, 560-562.

[4] Altieri M, Santangelo G (2021) The psychological impact of COVID19 pandemic and lockdown on caregivers of people with dementia. Am J Geriatr Psychiatry 29, 27-34.

[5] Dang S, Penney LS, Trivedi R, Noel PH, Pugh MJ, Finley E, Pugh JA, Van Houtven CH, Leykum L (2020) Caring for caregivers during COVID-19. J Am Geriatr Soc 68, 21072201.

[6] Muhammad A, Prince HA (2020) Caring for dementia caregivers in times of the COVID-19 crisis: A systematic review. Am J Nurs Resh 8, 552-561.

[7] Wang H, Li T, Barbarino P, Gauthier S, Broday H, Molinuevo JL, Xie H, Sun Y, Yu E, Tang Y, Weidner W, Yu X (2020) Dementia care during COVID-19. Lancet 395, 1190-1191.

[8] Cagnin A, Di Lorenzo R, Marra C, Bonanni L, Cipidi C, Laganà V, Rubino E, Vacca A, Provero $P$, Isella V, Vanacore N, Agosta F, Appollonio I, Caffarra P, Pettenuzzo I, Sambati R, Quaranta D, Guglielmi V, Logroscino G, Filippi M, Tedeschi G, Ferrarese C, Rainero I, Bruni AC, SINDem COVID-19 study group (2020) Behavioral and psychological effects of coronavirus disease-19 quarantine in patients with dementia. Front Psyhiatry 11, 578015.
[9] Rainero I, Bruni AC, Marra C, Cagnin A, Bonanni L, Cupidi C, Laganà V, Rubino E, Vacca A, Di Lorenzo R, Provero P, Isella V, Vanacore N, Agosta F, Appollonio I, Caffarra $P$, Bussè C, Sambati R, Quaranta D, Guglielmi V, Logroscino G, Filippi M, Tedeschi G, Ferrarese C, SINdem COVID-19 Study Group (2021) The impact of COVID-19 quarantine on patients with dementia and family caregivers: A nation-wide survey. Front Aging Neurosci 12, 625781 .

[10] Gaugler JE, Mittelman MS, Hepburn K, Newcomer R (2009) Predictors of change in caregiver burden and depressive symptoms following nursing home admission. Psychol Aging 24, 385-396.

[11] Carpinelli Mazzi M, Iavarone A, Musella C, De Luca M, De Vita D, Branciforte S, Coppola A, Scarpa R, Raimondo S, Sorrentino S, Lualdi F, Postiglione A (2020) Time of isolation, education and gender influence the psychological outcome during COVID-19 lockdown in caregivers of patients. Eur Geriatr Med 11, 1095-1098.

[12] Bressan V, Visintini C, Palese A (2020) What do family caregivers of people with dementia need? A mixed-method systematic review. Rev Health Soc Care Community 28, 1942-1960.

[13] Arighi A, Fumagalli GG, Carandini T, Pietroboni AM, De Riz MA, Galimberti D, Scarpini E (2021) Facing the digital divide into a dementia clinic during COVID-19 pandemic: Caregiver age matters. Neurol Sci 42, 1247-1251.

[14] Beaunoyer E, Dupéré S, Guitton MJ (2020) COVID-19 and digital inequalities: Reciprocal impacts and mitigation strategies. Comput Human Behav 111, 106424.

[15] Cummings JL, Mega M, Gray K, Rosenberg-Thompson S, Carusi DA, Gornbein J (1994) The Neuropsychiatric Inventory: Comprehensive assessment of psychopathology in dementia. Neurology 44, 2308-2314.

[16] Chattat R, Cortesi V, Izzicupo F, Del Re ML, Sgarbi C, Fabbo A, Bergonzini E (2011) The Italian version of the Zarit Burden interview: A validation study. Int Psychogeriatr 23, 797-805.

[17] Bianchetti A, Cornali C, Ranieri P, Trabucchi M (2017) Quality of life in patients with mild dementia. Validation of the Italian version of the quality of life Alzheimer's disease (QoL-AD) Scale. J Gerontol Geriatr 65, 137-143.

[18] Weiss DS, Marmar, CR (1996) The Impact of Event Scale - Revised. In Assessing psychological trauma and PTSD, Wilson J, Keane TM, eds. Guilford, New York, pp. 399-411.

[19] Folstein MF, Folstein SE, McHugh PR (1975) 'Mini-Mental State'. A practicle method for grading the cognitive state of patients for the clinician. J Psychiatr Res 12, 189-198.

[20] Lam K, Lu AD, Shi Y, Covinsky KE (2020) Assessing telemedicine unreadiness among older adults in the United States during the COVID-19 pandemic. JAMA Intern Med 180, 1389-1391.

[21] Yoon H, Jang Y, Vaughan PW, Garcia M (2020) Older adults' internet use for health information: Digital divide by race/ethnicity and socioeconomic status. J Appl Gerontol 39, 105-110.

[22] Alzheimer Europe. Living with dementia: COVID19 URL: https://www.alzheimer-europe.org/Living-withdementia/ COVID-19 [accessed 2020-05-14],

[23] Cotterell N, Buffel T, Phillipson C (2018) Preventing social isolation in older people. Maturitas 113, 80-84.

[24] Smith AC, Thomas E, Snoswell CL, Haydon H, Mehrotra A, Clemensen J, Caffery LJ (2019) Telehealth for global emergencies: Implications for coronavirus disease 2019 (COVID-19). J Telemed Telecare 26, 309-313. 
[25] Goodman-Casanova JM, Dura-Perez E, Guzman-Parra J, Cuesta-Vargas A, Mayoral-Cleries F (2020) Telehealth home support during COVID-19 confinement for community-dwelling older adults with mild cognitive impairment or mild dementia: Survey study. J Med Internet Res 22, e19434.

[26] Boots LMM, De Vugt ME, Van Knippenberg RJM, Kempen GIJM, Verhey FRJ (2014) A systematic review of Internetbased supportive interventions for caregivers of patients with dementia. Int J Geriatr Psychiatry 29, 331-344.

[27] Cristancho-Lacroix V, Wrobel J, Cantegreil-Kallen I, Dub T, Rouquette A, Rigaud AS (2015) A web-based psychoeducational program for informal caregivers of patients with Alzheimer's disease: A pilot randomized controlled trial. $J$ Med Internet Res 17, e117.

[28] Egan KJ, Pinto-Bruno ÁC, Bighelli I, Berg-Weger M, van Straten A, Albanese E, Pot AM (2018) Online training and support programs designed to improve mental health and reduce burden among caregivers of people with dementia: A systematic review. J Am Dir Assoc 19, 200-206.

[29] Guay C, Auger C, Demers L, Mortenson WB, Miller WC, Gśelinas-Bronsard D, Ahmed S (2017) Components and outcomes of internet-based interventions for caregivers of older adults: Systematic review. JMed Internet Res 19, e313.

[30] Hurley RVC, Patterson TG, Cooley S J (2014) Meditationbased interventions for family caregivers of people with dementia: A review of the empirical literature. Aging Ment Health 18, 281-288.

[31] Jensen M, Agbata IN, Canavan M, McCarthy G (2015) Effectiveness of educational interventions for informal caregivers of individuals with dementia residing in the community: Systematic review and meta-analysis of randomised controlled trials. Int J Geriatr Psychiatry 30, 130-143.

[32] Aledeh M, Kotera Y (2020) Research Proposal: Qualitative investigation into Internet-based interventions for professional dementia caregivers' wellbeing. J Concurrent Disord, pp. 1-10.

[33] Waller A, Dilworth S, Mansfield E, Sanson-Fisher R (2017) Computer and telephone delivered interventions to support caregivers of people with dementia: A systematic review of research output and quality. BMC Geriatr 17, 265. 